(1]

Dept of Respiratory Medicine, Norfolk and Norwich University Hospital, Norwich, UK.

\title{
A 31-year-old female with a rare cause of recurrent lower lobar collapses
}

\section{Case report}

Cite as: Banka R, Terrington D, Kamath AV. A 31-year-old female with a rare cause of recurrent lower lobar collapses. Breathe 2018; 14: e72-e77.
A 31-year-old female presented to the emergency department with a 5-day history of feeling generally unwell, with a sore throat, breathlessness and a productive cough with expectoration of clear mucoid sputum. Her exercise tolerance, previously unlimited, had declined to approximately $90 \mathrm{~m}$. She denied any pyrexia, rigors, haemoptysis or night sweats.

Her past medical history included mild asthma, diagnosed following a positive histamine challenge test in 2015, for which she was not taking any regular medication. On direct questioning she had been admitted to hospital three times over the course of 4 years with chest infections, all of which had resulted in collapse of the left lower lobe and improved radiologically after treatment with chest physiotherapy, nebulisers and antibiotics (figure 1). There was no history of recurrent childhood infections, steatorrhoea, otitis media or weight loss. Systemic enquiry was otherwise unremarkable.

She was allergic to clarithromycin and was not taking any regular medications.

In terms of social history, she lived at home with her husband and three children all of whom were fit and healthy. She had a trivial smoking history of 3 pack-years and denied any significant alcohol consumption. There was no history of recent foreign travel and no significant family history, in particular cystic fibrosis.

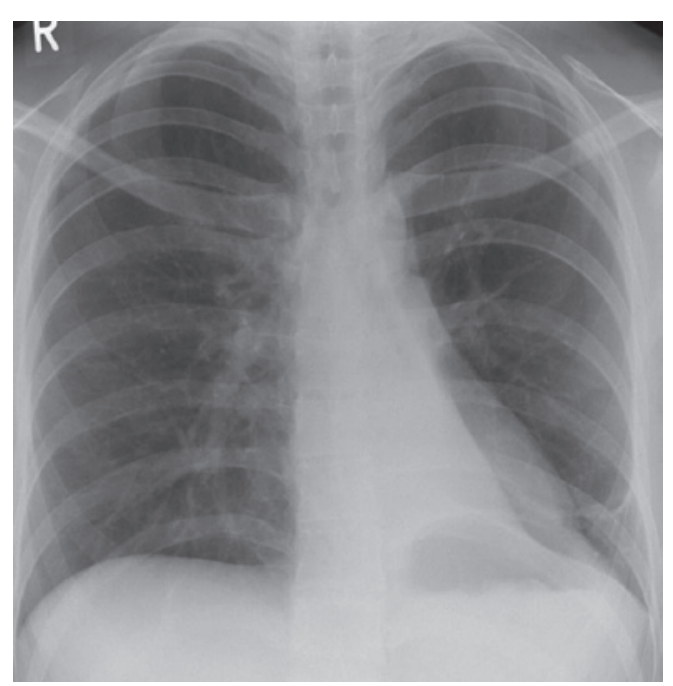

Figure 1 Chest radiograph demonstrating left lower lobe collapse during a previous admission, which improved following treatment with antibiotics, nebulisers and chest physiotherapy.

On examination she was tachycardic with a heart rate of 110 beats per min and tachypnoeic with a respiratory rate of 28 breaths per min. Oxygen saturations were $92 \%$ on $60 \%$ oxygen (venture mask). Her blood pressure was maintained at $120 / 68 \mathrm{mmHg}$ and she was 
apyrexial. She was in obvious respiratory distress with the use of her accessory muscles. There was no evidence of pallor, clubbing or stigmata of systemic disease. There were no cardiac murmurs on auscultation. Auscultation of her chest revealed reduced breath sounds bilaterally with dullness to percussion.

\section{Task 1}

Which of the following is the least likely diagnosis?
a) Lung collapse
b) Pneumonia
c) Asthma exacerbation
d) Interstitial lung disease 


\section{Answer 1}

d) Interstitial lung disease

On admission an arterial blood gas (ABG) on $60 \%$ oxygen demonstrated severe type 1 respiratory failure with a $\mathrm{pH}$ of 7.46 , carbon dioxide tension of $4.48 \mathrm{kPa}$, oxygen tension $\left(\mathrm{PO}_{2}\right)$ of $8.28 \mathrm{kPa}, \mathrm{HCO}_{3}{ }^{-}$ of $24.5 \mathrm{mmol} \cdot \mathrm{L}^{-1}$, base excess of $0.0 \mathrm{mmol} \cdot \mathrm{L}^{-1}$ and oxygen saturations of $91 \%$. Lactate was $0.9 \mathrm{mmol} \cdot \mathrm{L}^{-1}$. An electrocardiogram revealed a sinus tachycardia and a portable chest radiograph was carried out (figure 2).

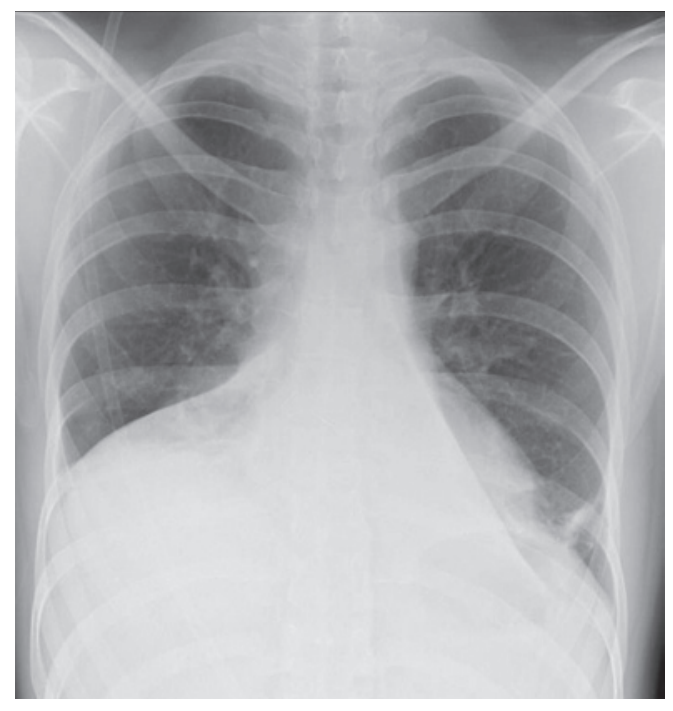

Figure 2 Chest radiograph on admission.

Task 2

What does the chest radiograph in Figure 2 demonstrate?

\section{Task 3}

Which of the following tests is/are the next step in the investigation?

a) Chest computed tomography (CT)

b) Allergic bronchopulmonary aspergillosis screen

c) Sputum culture 


\section{Answer 2}

Bilateral lower lobe collapse.

\section{Answer 3}

$\mathrm{a}-\mathrm{c}$ ) They are all appropriate next steps in the investigation.

In view of the degree of hypoxaemia and the chest radiograph findings an urgent highresolution CT scan of the thorax was organised, which confirmed bilateral lower lobe collapse and partial collapse and consolidation of the right middle lobe. No obstructing endobronchial lesion was evident and there was no hilar or mediastinal lymphadenopathy (figure 3).

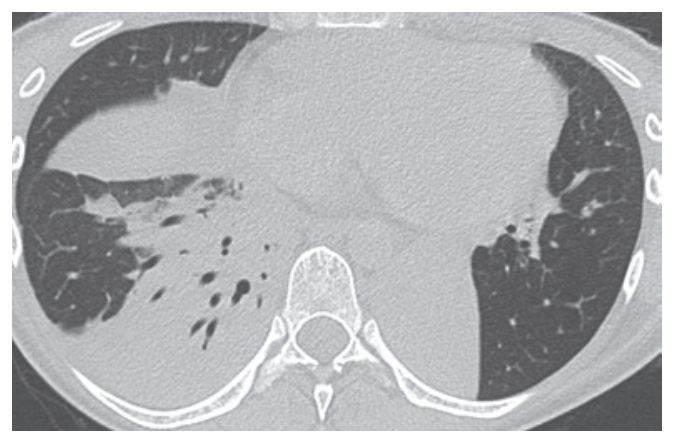

Figure 3 High-resolution CT scan demonstrating bilateral lobe collapses with consolidation in the right middle lobe and partial collapse.

\section{Task 4}

Which of the following is/are the causes of lobar lung collapse?
a) Endobronchial tumour
b) Lymph node compression on the bronchus
c) Allergic bronchopulmonary aspergillosis
d) Cystic fibrosis 


\section{Answer 4}

a-d) They are all causes of lobar lung collapse.

Admission bloods showed a raised white cell count and neutrophilia of $15.6 \times 10^{9} \cdot \mathrm{L}^{-1}$ and $15.2 \times 10^{9} \cdot \mathrm{L}^{-1}$, respectively. C-reactive protein was slightly elevated at $30 \mathrm{mg} \cdot \mathrm{L}^{-1}$. Remaining baseline bloods, including full blood count, urea and electrolytes, and liver function tests all remained within normal parameters. Total IgE was normal at $43 \mathrm{kU} \cdot \mathrm{L}^{-1}$, eosinophil count was $0.0 \times 10^{9} \cdot \mathrm{L}^{-1} \cdot \operatorname{lgG}$ levels to Aspergillus were normal at $21.9 \mathrm{mg} \cdot \mathrm{L}^{-1}$ and radioallergosorbent test to Aspergillus fumigatus and Aspergillus niger was undetectable. Anti-neutrophil cytoplasmic antibody and anti-nuclear antibody screens were negative, as was a sweat test with a chloride of $23 \mathrm{mmol} \cdot \mathrm{L}^{-1}$. Two sputum cultures revealed normal respiratory flora with no significant bacterial growth. She responded well to chest physiotherapy, antibiotics and nebulisers and her repeat $A B G$ showed a $P_{2}$ of $10.28 \mathrm{kPa}$ on room air and she was subsequently discharged.

She underwent a bronchoscopy as an outpatient and it excluded any endobronchial lesions and demonstrated widespread near collapse of the main bronchi upon expiration and gentle suctioning, confirming bronchomalacia (figures 4 and 5). There was no evidence of concurrent tracheomalacia during bronchoscopy or during the expiratory phase of the high-resolution CT scan. Bronchoalveolar lavage from the lower lobes was negative for bacterial organisms, yeasts, fungi and acid-alcohol-fast bacilli. Cytology demonstrated a mixed population of inflammatory cells comprised of respiratory epithelial cells, alveolar macrophages and neutrophils. The flow-volume loop was also consistent with variable intrathoracic airflow obstruction (figure 6).

She remained symptomatically well and her lung function tests remained within normal parameters. Lung functions showed a forced expiratory volume in $1 \mathrm{~s}$ (FEV1)/forced vital capacity (FVC) of $81.97 \%$, FEV1 of $3.20 \mathrm{~L}$ (91\% predicted)

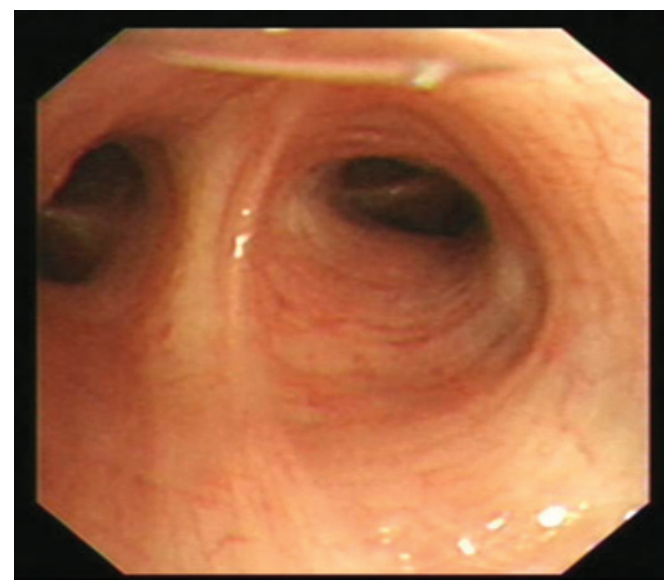

Figure 4 Left upper lobe orifice during inspiration on bronchoscopy.

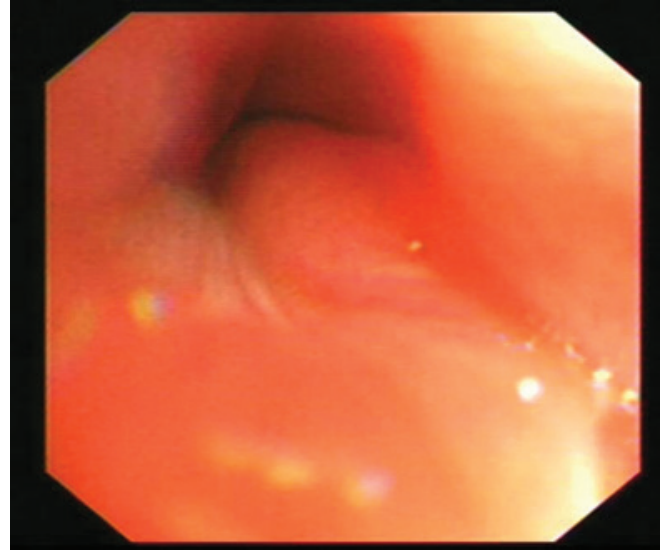

Figure 5 Collapse of the left upper lobe during expiration on bronchoscopy, confirming bronchomalacia.

and FVC of $3.90 \mathrm{~L}$ (97\% predicted). A repeat chest radiograph confirmed complete resolution of the previously demonstrated lobar collapses. In view of the previous positive histamine challenge test, she was commenced on inhaled corticosteroids and given the intermittent nature of her symptoms she was not offered domiciliary noninvasive ventilation.

Bronchomalacia is a defined as a defect of the composition of the cartilage of the bronchi, which results in loss of mechanical integrity and resistance of the airway during expiration [1]. As such the lumen of the airway is narrowed during expiration and more than a 50\% reduction in the diameter of the lumen is pathognomonic of bronchomalacia [2]. The condition can affect any part of the bronchial tree and may occur in isolation or in conjunction with the trachea resulting in tracheobronchomalacia. It can be classified as congenital, occurring during infancy which may be an isolated finding, or may be acquired during adulthood due to damage to the bronchial tree. Such insults include chronic infection, tuberculosis, trauma, chronic bronchitis, post-intubation, thoracic malignancies and amyloidosis [1, 3, 4].

Symptoms can often overlap and masquerade as many other common respiratory conditions with shortness of breath, wheeze, difficulty in clearing secretions and recurrent chest infections. There are several cases of bronchomalacia presenting as difficult to control asthma [1, 5].

Bronchoscopy is the gold standard in order to confirm a diagnosis of bronchomalacia as it allows real time visualisation and characterisation of the airways. It can be used in conjunction with radial endobronchial ultrasound or optical coherence tomography. Bronchomalacia can be classified as mild, moderate or severe depending upon the extent of bronchial collapse during expiration on bronchoscopy [6]. A decrease in the size of the lumen by $\leq 50 \%$ during exhalation is regarded normal, between $51 \%$ and $75 \%$ as mild obstruction, between $76 \%$ and $90 \%$ as moderate obstruction, and between $91 \%$ and $100 \%$ as severe 
obstruction [7]. Other modalities to diagnose bronchomalacia include dynamic airway computed tomography [8]. Pulmonary function tests in bronchomalacia may show an obstructive pattern with diminished expiratory flow and notching of the flow-volume loop; however, these findings are more pronounced with associated tracheomalacia as compared to isolated bronchomalacia [9].

Treatment options depend on the severity of symptoms, degree of airway collapse and underlying aetiology. Physiotherapy, noninvasive ventilation, surgery and stenting are used: all modalities aimed at preventing bronchial collapse. Physiotherapy includes pursed lip breathing and breathing through a resistor device. Use of noninvasive ventilation, in the form of intermittent continuous positive airway pressure (CPAP) during the day and overnight CPAP, can be used to stabilise the airways and also serve as a bridge to other definitive treatment options such as surgery or stents. Silicone or metal stents can be used in severe cases. However, it is associated with side-effects such as migration, occlusion due to granulation tissue or mucus plugs. Surgery is reserved for localised bronchomalacia when stenting has failed and includes procedures to reshape and reconstruct the airway wall and use of external stents [10].

In conclusion, although patients typically present with bronchomalacia during early childhood it can rarely be diagnosed in adulthood. The gold

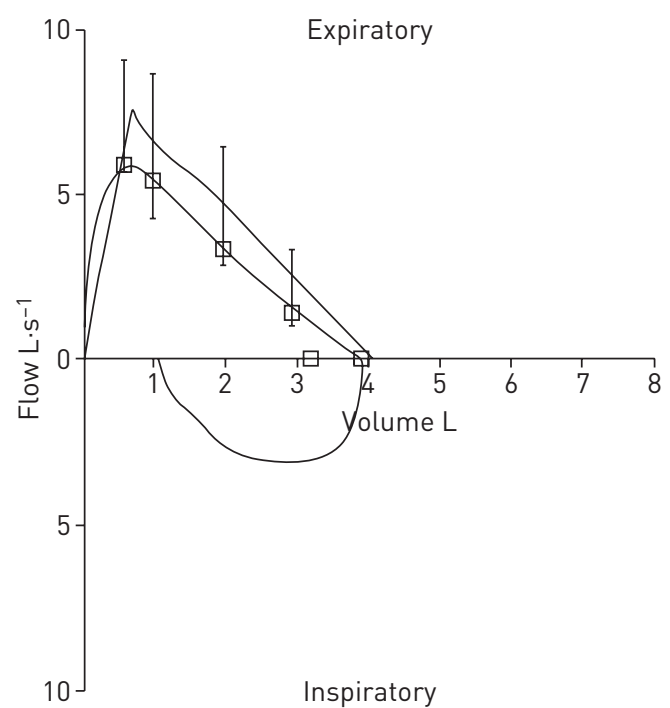

Figure 6 Flow-volume loop showing variable intrathoracic airflow obstruction.

standard to confirm a diagnosis is flexible fibreoptic bronchoscopy with a characteristic finding of a reduction in the lumen diameter $>50 \%$ during expiration. Bronchomalacia, thus, should be considered in the differential diagnosis for any patient presenting with recurrent lobar collapses.

\section{Conflict of interest}

None declared.

\section{References}

1. Sala A, Deltoro AM, Moragon EM. An asthmatic patient with bronchomalacia and good response with continuous airway pressure. Arch Bronconeumol 2014; 50: 202-208.

2. Nuutinen J. Acquired bronchomalacia. Eur J Respir Dis 1982; 63: 380-387.

3. Johnson TH, Mikita JJ, Wilson RJ, et al. Acquired tracheomalacia. Radiology 1973; 109: 577-580.

4. Uyar M, Sanli M, Kervancioglu S, et al. Tracheobronchomalacia as a rare cause of chronic dyspnoea in adults. Med Princ Pract 2017; 26: 179-181.

5. Andregnette MV, de la Barrera EH, Flandes-Aldeyturriaga J, et al. Resolution of bronchomalacia presenting as severe asthma by endoscopic intervention. Ann Allergy Asthma Immunol 2011; 106: 443-444.
6. García-Donas J, Nunez MJ, Fernández JM, et al. Recurrent pneumonia is secondary to idiopathic bronchomalacia. Arch Bronconeumol 2001; 37: 324-325.

7. Ernst A, Majid A, Feller-Kopman D, et al. Airway stabilization with silicone stents for treating adult tracheobronchomalacia: a prospective observational study. Chest 2007; 132: 609-616.

8. Lee KS, Sun MR, Ernst A, et al. Comparison of dynamic expiratory CT with bronchoscopy for diagnosing airway malacia: a pilot evaluation. Chest 2007; 131: 758-764.

9. Murgu SD, Colt HG. Tracheobronchomalacia and excessive dynamic airway collapse. Respirology 2006; 11: 388-406.

10. Murgu SD, Colt HG. Treatment of adult tracheobronchomalacia and excessive dynamic airway collapse: an update. Treat Respir Med 2006; 5: 103-115. 